\title{
Desarrollar estrategias didácticas para la adquisición de competencias investigativas en estudiantes de octavo grado del Centro de Investigación e Innovación Educativas
}

\section{Developing teaching strategies for the acquisi- tion of research competences in eighth graders of the Educational Research and Innovation Center}

\author{
Sonia Patricia Guity López \\ sguity@upnfm.edu.hn \\ José Alfonzo Mendoza Corrales ${ }^{* *}$ \\ jmendoza@upnfm.edu.hn
}

\section{Resumen}

Este artículo muestra el resultado de la intervención realizada en el octavo grado de Educación Básica del Centro de Investigación e Innovación Educativas (CIIE) de la Universidad Pedagógica Nacional Francisco Morazán (UPNFM) en donde mediante un
Diseño No Experimental, (pretest y postest) se implementaron diversas estrategias didácticas en Matemáticas, Ciencias Naturales y Ciencias Sociales, para desarrollar competencias investigativas en los estudiantes. Los resultados de la Anova, aplicada en el Postest,

La investigación "Desarrollar estrategias didácticas para la adquisición de competencias investigativas en estudiantes de octavo grado del centro de investigación e innovación educativas," con número de subvención "FAI-001-2013" ha sido desarrollada con el financiamiento del Fondo de Apoyo a la Investigación de la Universidad Pedagógica Nacional Francisco Morazán de Honduras.

- Docente de la Universidad Pedagógica Nacional Francisco Morazán, Honduras.

* Docente de la Universidad Pedagógica Nacional Francisco Morazán, Honduras.

Recibido 23 de marzo de 2018 / Aceptado 24 de abril de 2018. 
Sonia Patricia Guity López, José Alfonzo Mendoza Corrales

evidenciaron que no hubo diferencias estadísticamente significativas entre los grupos control y experimental, sin embargo, permitió identificar que los estudiantes de octavo grado poseen primero competencias actitudinales, en segundo lugar, las procedimentales y finalmente, competencias conceptuales. La investigación realizada abre la posibilidad de continuar indagando sobre condiciones diversas del aula, docentes, centro educativo y tiempo de intervención, para lograr diferencias significativas en los resultados.

Palabras clave: competencias investigativas, estrategias didácticas, estudiantes, octavo grado

\section{Abstract}

This article shows the results of the intervention carried out in the eighth grade of Basic Education of the Center for Educational Research and Innovation (CIIE) of the Universidad Pedagógica Francisco Morazán (UPNFM) where, through a Non-Experimental Design (pretest and posttest) Implemented several didactic strategies in Mathematics, Natural Sciences and Social Sciences, to develop research competences in students, the results of the Anova applied in the
Postest evidenced that there were no statistically significant differences between the control and experimental groups, however, it allowed to identify that The eighth grade students, first possess attitudinal competences, second, procedural and finally, conceptual competences. The research opens the possibility of continuing to investigate diverse classroom conditions, teachers, educational center and intervention time to achieve significant differences in results.

Keywords: research competences, teaching strategies, students, eight grade 
Desarrollar estrategias didácticas para la adquisición de competencias investigativas

en estudiantes de octavo grado del Centro de Investigación e Innovación Educativas

\section{Introducción}

El artículo presenta el trabajo realizado en torno al proyecto de investigación Desarrollar estrategias didácticas para la adquisición de competencias investigativas en estudiantes de octavo grado del centro de investigación e innovación educativas, dicho proyecto fue realizado durante el año 2016 contando con el apoyo de los docentes titulares y en práctica de las asignaturas de Matemáticas, Ciencias Sociales y Ciencias Naturales, la investigación comenzó con la aplicación de una lista de chequeo como Pretest, auto administrada a cada estudiante; después de tres meses de intervención, se aplicó la misma lista para que los estudiantes determinarán si habían logrado o no alcanzar el conjunto de competencias conceptuales, procedimentales y actitudinales establecidas por el equipo de investigación y los docentes titulares de las correspondientes asignaturas.

Es importante señalar que la investigación continúa la línea de trabajo desarrollada en el centro desde el año 2015, en donde se realizaron dos trabajos de tesis en el marco de la maestría de Investigación educativa. Los resultados alcanzados en esa fecha revelan la necesidad de que los niños y niñas puedan alcanzar competencias investigativas, desde los primeros años de formación, respondiendo de esta forma a la exigencia del CNB por un lado, y por otro, las demandas de la sociedad actual que requiere cada vez más el empoderamiento y evidencia de competencias, que le permitan resolver asertivamente, distintas situaciones conflictivas personales y comunitarias. El artículo explica también los antecedentes, la justificación y el marco teórico que se configuraron para circunscribir la investigación. El texto finaliza con los principales hallazgos y conclusiones encontrados, producto de la Anova y la observación no participante utilizados como metodología.

\section{Discusión Teórica}

El panorama actual de la investigación en Honduras refleja que la actividad científica es reciente, prácticamente se remonta al cercano 
siglo XX, comenzando como un esfuerzo individual como respuesta a necesidades de generar nuevos conocimientos que contribuyeran al desarrollo social y económico del país; esta situación ha provocado poca producción científica agravada por la falta de recursos humanos y financieros. El desarrollo de la investigación está respaldada únicamente por tres artículos de la Constitución de la República de Honduras, el título III De las Declaraciones, Derechos y Garantías, Capítulo VIII de la Educación y la Cultura, artículos 155,160 y 175, destacándose en ellos la promoción y apoyo a la investigación realizada por centros extranjeros y nacionales.

No obstante esta expresa declaración, el apoyo dado a la investigación es muy escaso y es reciente el compromiso formal del gobierno con la ciencia y la tecnología. A partir de 1990 se destinaron fondos hacia este sector, se creó y organizó el Consejo Hondureño de Ciencia y Tecnología (COHCYT), hoy llamado IHCIETY, que otorga becas de estudio para postgrado en el país y en el extranjero, financia proyectos de investigación y publica revistas de circulación nacional sobre temas científicos y tecnológicos. (Suazo, 2002, p.3)

El Centro de Investigación e Innovación Educativas (CIIE), perteneciente a la U.P.N.F.M, es uno de los espacios académicos más propicios para fomentar, desde las bases, las destrezas para el desarrollo de la investigación, no como actividad o proyecto limitado a un tiempo o espacio específico, sino como un semillero permanente para la formación de pequeños investigadores que puedan afrontar exitosamente las demandas de la nueva sociedad del conocimiento, planteándose constantemente preguntas sobre su entorno cultural, social, político y económico y buscando respuestas reflexionadas a partir de una investigación ligada a contenidos, actividades educativas y formas de evaluación en las distintas asignaturas de su plan de estudios.

Tomando como punto de partida las experiencias exitosas anteriores de 2005 y 2007, se plantea ahora la necesidad de extender la experiencia al tercer ciclo de la educación básica. Específicamente el estudio se realizó en el octavo grado de ambas jornadas, con la particularidad que en el matutino los estudiantes provienen en su

34 Instituto de Investigación y Evaluación Educativas y Sociales / Universidad Pedagógica Nacional Francisco Morazán Los artículos de la Revista Electrónica Paradigma del Instituto de Investigación y Evaluación Educativas y Sociales de la Universidad Pedagógica Nacional Francisco Morazán, se comparten bajo términos de la Licencia Creative Commons: Se permite que otros puedan escargar las obras y compartirlas con otras personas, siempre y cuando se reconozca su autoría, pero no se pueden cambiar de $Y$ NC ND ninguna manera ni se pueden utilizar comercialmente. 
mayoría de la misma institución, en cambio el octavo vespertino, recibe a varios estudiantes de otras instituciones públicas y privadas del país mediante un examen de admisión; por lo que es además, la oportunidad de establecer las fortalezas y debilidades en la formación de competencias investigativas en los dos grupos de estudiantes participantes.

El Currículo Nacional Básico (CNB), plantea muchas ideas que pudieran transformarse en estrategias didácticas para formar o fortalecer competencias investigativas, sin embargo, una gran parte de las instituciones de educación básica siguen guiándose en su currículo por los libros de textos, programas diseñados para ciertos centros educativos, u otras formas de planificación didáctica, que no permiten un apoyo sistemático a la política estatal del CNB; se requiere entonces buscar alternativas viables que puedan compartirse con las instituciones públicas y privadas interesadas en proporcionar una educación integral y de calidad.

Para hablar de competencias investigativas es importante su definición. El término proviene del latín competens, competentis (Moliner, 1998, citado por Muñoz Giraldo y otros, 2001, p.15) el que tiene aptitud legal o autoridad para resolver cierto asunto o también el que conoce, es experto o apto en cierta ciencia o materia. LevyLeboyer (1996), citado por Balbo (s/f) define las competencias como tareas o situaciones de trabajo, conjunto de conductas organizadas en el seno de una estructura mental, relativamente estables y movilizables cuando es preciso; las vincula con una actividad, es por eso que la persona competente moviliza el saber en el momento oportuno, sin tener necesidad de consultar reglas básicas, ni de preguntarse sobre las indicaciones de tal conducta.

Desarrollar estas competencias, actitudes y valores para formar investigadores, no es una tarea fácil, sin embargo, la historia educativa registra ya experiencias y proyectos concretos (Colombia, Venezuela, México, Argentina...) en donde la implementación de varias estrategias ha conllevado exitosamente a este logro. De igual forma, estas competencias pueden englobarse dentro de una denominación que clasifica las competencias en conceptuales, procedimentales y actitudinales.

(1) $\$$ Los artículos de la Revista Electrónica Paradigma del Instituto de Investigación y Evaluación Educativas y Sociales de la Universidad Pedagógica Nacional Francisco Morazán, se comparten bajo términos de la Licencia Creative Commons: Se permite que otros puedan descargar las obras y compartirlas con otras personas, si 
Sonia Patricia Guity López, José Alfonzo Mendoza Corrales

\section{Competencias conceptuales}

Estas competencias se evidencian en la adquisición de conceptos y nociones fundamentales que le permitan a los niños y niñas, conocer y comprender el mundo cotidiano y científico: plantear hipótesis y buscar posibles soluciones a los problemas propuestos. Se establecieron ocho competencias en esta clasificación.

\section{Competencias procedimentales}

Estas habilidades implicaron la adquisición y aplicación de distintos procedimientos, para obtener y procesar la información relacionada con los problemas propuestos en clases o en el proceso de investigación. Para este grupo se seleccionaron 13 competencias.

\section{Competencias actitudinales}

Estas competencias involucraron los valores personales y colectivos, de los cuales se apropiaron los y las estudiantes para comenzar a modelar su espíritu científico como investigador o investigadora. Para este tipo de competencias se definieron seis competencias.

\section{Estrategias didácticas}

Para el desarrollo de las competencias investigativas descritas anteriormente, se partió del concepto de estrategias didácticas como un conjunto de Acciones planificadas por el docente, con el objetivo de que el estudiante logre la construcción del aprendizaje y se alcancen los objetivos planteados. Una estrategia didáctica es, en un sentido estricto, un procedimiento organizado, formalizado y orientado a la obtención de una meta claramente establecida (UNED, s/f).

Esta definición implica varios elementos valiosos que fueron considerados en la investigación, lo primero es que se trata de acciones planificadas, no surgidas o realizadas de manera espontánea; en este sentido, los docentes participantes del estudio seleccionaron cuidadosamente varias estrategias que condujeran a la concreción

36 Instituto de Investigación y Evaluación Educativas y Sociales / Universidad Pedagógica Nacional Francisco Morazán

Los artículos de la Revista Electrónica Paradigma del Instituto de Investigación y Evaluación Educativas y Sociales de la Universidad
Pedagógica Nacional Francisco Morazán, se comparten bajo términos de la licencia Creative Commons: Se permite eque otros (i) $-\left(\begin{array}{l}\text { Pedagógica Nacional Francisco Morazán, se comparten bajo términos de la Licencia Creative Commons: Se permite que otros puedan } \\ \text { descargar las obras y compartillas con otras personas, siempre y cuando se reconozca su autoría, pero no se pueden cambiar de }\end{array}\right.$ Percialmente. 
de las competencias definidas preliminarmente. En segundo lugar, el propósito de la selección de estrategias, es lograr un aprendizaje significativo; en este sentido, las estrategias que se implementaron en la investigación constituyen un caudal de experiencia acumulada por los docentes del CIIE, en particular en el área de Ciencias Naturales. Por otro lado, las estrategias didácticas correspondieron a la coherencia y pertinencia de los temas vinculados al desarrollo de las competencias investigativas.

Algunas de las estrategias implementadas fueron: En Ciencias Naturales, la Hoja de cálculo JOMAR (por el autor, docente del CIIE, José Manuel Ramírez) y Noche familiar científica. En Ciencias Sociales, El Afiche Personalizado, Mi Primera Investigación científica y Mi Menú Histórico. En Matemáticas, Pluto y el hueso, Práctica de laboratorio, con el programa Geógebra y el Bingo fueron las estrategias que más motivación causaron entre los estudiantes, evidenciando que mediante el juego pueden lograrse aprendizajes significativos.

\section{Objetivos del estudio}

\section{Objetivo general}

Implementar estrategias didácticas para desarrollar competencias investigativas en estudiantes de octavo grado de ambas jornadas en el Centro de Investigación e Innovación Educativas.

\section{Objetivos específicos}

- Identificar el tipo y nivel de competencia investigativa que poseen los estudiantes inscritos en el octavo grado del CIIE, a través de la aplicación de un pretest y postest diseñado en la investigación.

- Realizar actividades de aprendizaje significativo vinculadas a las estrategias didácticas que desarrollen competencias investigativas en los estudiantes participantes.

- Determinar las estrategias didácticas más significativas para 
Sonia Patricia Guity López, José Alfonzo Mendoza Corrales

el aprendizaje de los estudiantes en las asignaturas seleccionadas.

- Describir las competencias investigativas más pertinentes para los estudiantes de octavo grado de educación básica luego de la experiencia desarrollada.

\section{Métodos y Materiales}

\section{Población}

Para efectos de este estudio, la población fue el total de los estudiantes de octavo grado del Centro de Investigación e Innovación Educativas de la Universidad Pedagógica Nacional “Francisco Morazán” del año 2016. Este grado contó con 78 estudiantes, divididos en dos jornadas, jornada matutina, 40 estudiantes, 17 niñas y 23 varones; jornada vespertina, 23 niñas y 15 varones.

\section{Muestra}

La selección de la muestra es intencionada, ya que se necesita la participación de todos los estudiantes matriculados en octavo grado, año 2016. La muestra fue de 78 estudiantes.

El diseño del estudio realizado fue un No experimental del tipo Cuasiexperimental, ya que se trabajó con grupos intactos, estableciendo comparaciones entre el grupo experimental (octavo vespertino) y el control (octavo matutino), el entorno de la investigación realizada fue el Centro de Investigación e Innovación Educativas (CIIE) de la Universidad Pedagógica Nacional Francisco Morazán (UPNFM).

\section{Técnicas de recolección de datos}

Tomando en consideración el enfoque utilizado en la investigación las técnicas consideradas más apropiadas son: 
Lista de chequeo

Se diseñó una lista de chequeo con las 27 competencias investigativas seleccionadas en el proyecto, para luego emplearla como Pretest (antes de la intervención) y postest (después de la intervención) a los dos grupos de estudiantes participantes de octavo grado del CIIE, Octavo matutino como grupo control y Octavo vespertino como grupo experimental, en esta lista de chequeo, se le pidió a cada estudiante, marcar con una $X$, si consideraba que poseía la competencia enlistada para 3 diferentes asignaturas: Matemáticas, Ciencias Sociales y Ciencias Naturales.

\section{Observación no participante}

Se empleó durante todo el período de intervención, para observar la forma en que los docentes que participaron en el estudio, desarrollaron las estrategias seleccionadas, cada observación fue registrada en Protocolos de observación para luego contrastarlo con los resultados cuantitativos obtenidos.

Técnicas de análisis de datos

Para analizar los datos recolectados a través de la Escala, se empleó la ANOVA, previo a realizar el análisis descriptivo de rigor, el propósito de la Anova será determinar las diferencias estadísticamente entre los grupos de estudiantes participantes en el estudio.

\section{Variables}

Competencias investigativas

Definición conceptual

Tareas o situaciones de trabajo, conjunto de conductas organizadas en el seno de una estructura mental, relativamente estables y movilizables cuando es preciso; las vincula con una actividad, es por eso que la persona competente moviliza el saber en el momento 
Sonia Patricia Guity López, José Alfonzo Mendoza Corrales

oportuno, sin tener necesidad de consultar reglas básicas, ni de preguntarse sobre las indicaciones de tal conducta.

\section{Definición operacional}

Habilidades y destrezas evidenciadas por los estudiantes del CIIE de octavo grado durante y al final del período de intervención.

Estrategias didácticas

Definición conceptual

Arte de coordinar distintas acciones de investigaciones conducentes a la formación de competencias investigativas.

Definición operacional

Actividad realizada en el aula de clases o fuera de ella, para la formación de competencias investigativas en los estudiantes de octavo grado del CIIE.

\section{Resultados}

En la investigación, se evaluaron mediante un Pretest y un Postest, 27 competencias, divididas en conceptuales, procedimentales y actitudinales. Las competencias conceptuales son: Propongo problemas en clase, Planteo posibles soluciones para resolver problemas, Hago preguntas sobre los temas desarrollados, Participo activamente en los temas desarrollados, Manejo más contenido que el abordado en clase, Explico conceptos o términos sobre los temas, Propongo soluciones a problemas surgidos en clase e Invento nuevos problemas. Las competencias procedimentales definidas fueron: Doy juicios de valor sobre los temas estudiados, Analizo textos escritos y orales, Busco información adicional sobre los temas, Relaciono mi entorno con los temas desarrollados, Comunico oralmente mis ideas, Elaboro informes de trabajo, Interpreto dibujos y gráficas, Registro observaciones a través de dibujos y gráficas, Utilizo la computadora

40 Instituto de Investigación y Evaluación Educativas y Sociales / Universidad Pedagógica Nacional Francisco Morazán

Los artículos de la Revista Electrónica Paradigma del Instituto de Investigación y Evaluación Educativas y Sociales de la Universidad (1) -1 Pedagógica Nacional Francisco Morazán, se comparten bajo términos de la Licencia Creative Commons: Se permite que otros puedan inguna manera ni se pueden utilizar comercialmente. 
como herramienta para la resolución de problemas, Realizo proyectos multidisciplinares utilizando la computadora, Utilizo enciclopedias y buscadores de la web para hacer investigaciones, Selecciono apropiadamente los materiales y equipos para realizar una demostración y Manejo objetos e instrumentos de laboratorio.

Las competencias actitudinales fueron: Acepto las sugerencias y/o aportes que me hacen mis compañeros y compañeras, Argumento mis respuestas, Colaboro con mi equipo cumpliendo mis asignaciones, Sigo instrucciones para realizar mis trabajos, Participo en actividades dentro y fuera del aula y Muestro curiosidad por cosas nuevas.

En el Pretest aplicado, la competencia de Propongo problemas en clase, estuvo particularmente posicionada en la clase de matemáticas en ambos grupos con un porcentaje de $29 \%$ del $100 \%$ de estudiantes consultados, esta asignatura logró la posición más alta, seguida de la opción de "todas las asignaturas", es decir, que para los estudiantes en las tres asignaturas poseen esta competencia, en tanto en el Postest, la ubicación más alta fue para Ciencias Sociales $(23 \%)$, seguido por Matemáticas(20\%); lo que indica que para los estudiantes de octavo grado, el desarrollo de esta competencia gira en torno a matemáticas $\mathrm{y}$ ciencias sociales.

En el Pretest, la competencia de Planteo posibles soluciones en clase, la asignatura que alcanzó la puntuación más alta en promedio fue Matemática (37\%), con un porcentaje bastante alto en comparación con las otras asignaturas en la intervención, en efecto, Iñiguez (2014) citando a Niss (2003) señala que Plantear y resolver problemas matemáticos, forma parte de las competencias involucradas en preguntar y responder sobre las matemáticas y a través de las matemáticas. En los resultados del postest, la asignatura de Matemáticas (20\%) alcanzó nuevamente, el porcentaje individual más alto, seguido por las 3 asignaturas en estudio (Ciencias Sociales, Ciencias Naturales y Matemáticas en conjunto), manifestando los estudiantes de octavo grado que la intervención desarrollada permitió posicionar en un mismo nivel.

La competencia de Hago preguntas sobre los temas desarrollados, los estudiantes señalaron que en las 3 asignaturas se les ha desarrollado, 
la habilidad de hacer preguntas sobre los temas desarrollados, con un porcentaje de $(40 \%)$, de manera individual, la asignatura de matemáticas logró un porcentaje considerable (15\%). En el Postest, de manera congruente con el resultado del Pretest, las 3 asignaturas alcanzaron el porcentaje más alto de $(43 \%)$, señalando que de manera coordinada, las estrategias realizadas en estas asignaturas, fomentaron el desarrollo de esta competencia.

Participo activamente en los temas desarrollados fue la cuarta competencia conceptual propuesta, en el Pretest, los estudiantes consideraron que tienen esta competencia más desarrollada en las 3 asignaturas, objeto de estudio, con un porcentaje importante de $34.2 \%$, en segundo lugar, de manera conjunta, las asignaturas de Ciencias Sociales y Ciencias Naturales con $14.5 \%$ respectivamente, es interesante señalar que de manera colectiva, las tres asignaturas fueron visualizadas como comunes para desarrollar esta competencia. Similares resultados se alcanzaron en el postest, donde las respuestas de los estudiantes evidenciaron que en las tres asignaturas han logrado esta competencia, con un porcentaje de $31 \%$.

La competencia de Manejo más contenido que el abordado en clase, en el Pretest para esta competencia se encontró que la mayoría de los estudiantes de octavo grado No poseen esta competencia con $26.3 \%$, mientras que en segunda instancia, otro grupo de estudiantes afirmó tener esta competencia en las tres asignaturas del estudio. De manera consistente y a pesar de la intervención realizada, los datos revelaron que los estudiantes, en el Postest consideran que No poseen esta competencia con un $27 \%$, más elevado que en el Pretest, ello implica, que los estudiantes manifiestan dominio de contenido impartido únicamente por los docentes, pero no de manera adicional.

Explico conceptos o términos sobre los temas fue la sexta competencia conceptual definida en este estudio, los estudiantes afirmaron que en el momento del Pretest no tienen esta competencia, manifestando esta afirmación con un $26 \%$ seguido por un alto porcentaje que afirmó tener dicha competencia en la clase de matemática, con un $20 \%$. De igual manera, en el Postest realizado, los estudiantes confirmaron que esta competencia no la tienen, casi con el mismo porcentaje del Pretest, un $27 \%$, seguido por la opción de las tres asignaturas. 
La séptima competencia de Propongo soluciones a problemas surgidos en clase, los estudiantes señalaron que en general tienen esta competencia en las tres asignaturas, objeto de estudio, con un $30 \%$. En el Postest, los resultados obtenidos fueron consistentes con el Pretest, ya que se logró un porcentaje bastante similar, con un $26 \%$, encontrándose solamente una diferencia porcentual de 4 puntos, la última competencia conceptual fue Invento nuevos problemas, la mayoría de los estudiantes manifestaron No poseer esta competencia en ninguna asignatura. De manera similar, los resultados del Postest señalaron que según el criterio de los estudiantes, todavía no han desarrollado esta competencia, con un porcentaje significativamente más alto que el Pretest, $38 \%$, este porcentaje estuvo seguido por la asignatura de matemáticas, siendo interesante evidenciar, que a pesar de que esta asignatura no estuvo situada en el primer lugar, al parecer es la disciplina, que promueve más competencias conceptuales.

De manera sintética, en los resultados del Postest aplicado a los estudiantes de Octavo grado del CIIE, puede evidenciarse que no existieron diferencias estadísticamente significativas entre los grupos (octavo matutino y vespertino), en las competencias conceptuales, señalando quizás nuevos caminos para profundizar en análisis posteriores, que puedan explicar, los factores o circunstancias que originaron estos resultados, factores como la intervención de docentes practicantes, estrategias implementadas, espacios físicos empleados para desarrollar las estrategias, tiempo de intervención, material didáctico utilizado, etc. como se muestra en la tabla 1:

\section{Tabla 1}

Resultados de anova en las competencias conceptuales

\begin{tabular}{llcc}
\hline Competencias Conceptuales & F & Sig. \\
\hline Propongo problemas en clase & $\begin{array}{c}\text { Entre grupos/Dentro } \\
\text { de grupos }\end{array}$ & 3.353 & .071 \\
$\begin{array}{l}\text { Planteo posibles soluciones para } \\
\text { resolver problemas }\end{array}$ & $\begin{array}{c}\text { Entre grupos/Dentro } \\
\text { de grupos }\end{array}$ & 2.235 & .139 \\
\hline
\end{tabular}




\begin{tabular}{lccc}
\hline \multicolumn{4}{c}{ Sonia Patricia Guity López, José Alfonzo Mendoza Corrales } \\
\hline $\begin{array}{l}\text { Hago preguntas sobre los temas } \\
\text { desarrollados }\end{array}$ & $\begin{array}{c}\text { Entre grupos/Dentro } \\
\text { de grupos }\end{array}$ & 1.968 & .165 \\
$\begin{array}{l}\text { Participó activamente en los temas } \\
\text { desarrollados }\end{array}$ & $\begin{array}{c}\text { Entre grupos/Dentro } \\
\text { de grupos }\end{array}$ & 1.653 & .203 \\
$\begin{array}{l}\text { Manejo más contenido que el } \\
\text { abordado en clase }\end{array}$ & $\begin{array}{c}\text { Entre grupos/Dentro } \\
\text { de grupos }\end{array}$ & .051 & .822 \\
$\begin{array}{l}\text { Explico conceptos o términos sobre } \\
\text { los temas }\end{array}$ & $\begin{array}{c}\text { Entre grupos/Dentro } \\
\text { de grupos }\end{array}$ & .234 & .630 \\
$\begin{array}{l}\text { Propongo soluciones a problemas } \\
\text { surgidos en clase }\end{array}$ & $\begin{array}{c}\text { Entre grupos/Dentro } \\
\text { de grupos }\end{array}$ & .006 & .936 \\
$\begin{array}{l}\text { Invento nuevos problemas } \\
\text { Entre grupos/Dentro } \\
\text { de grupos }\end{array}$ & .288 & .593 \\
\hline
\end{tabular}

Los resultados de las competencias procedimentales fueron diversos, es decir, que algunas de estas competencias lograron alcanzarse en una o varias asignaturas del estudio pero tres de ellas no lograron adquirirse. La primera competencia evaluada fue Doy juicios de valor sobre los temas estudiados, los estudiantes señalaron en los resultados del Pretest aplicado, que No poseen esta competencia en ninguna de las asignaturas propuestas en el estudio, con un porcentaje de $42 \%$. En el Postest aplicado, se encontró que los estudiantes opinaron también que esta competencia procedimental No ha sido desarrollada, casi con el mismo porcentaje del Pretest con un $41 \%$, este hecho, puede indicar que se necesitan implementar más estrategias o quizás debe considerarse, un tiempo más prolongado de intervención.

En la competencia de Analizo textos escritos y orales, los estudiantes indicaron que consideran que en las tres asignaturas se ha trabajado en la adquisición de esta competencia, con un $45 \%$, un porcentaje importante, en el Postest aplicado, de manera similar, los estudiantes encontraron que en las tres asignaturas, les ha permitido el análisis de textos escritos y orales, con un porcentaje de $38 \%$. Las estrategias de Investigación bibliográfica, Mapa conceptual, trabajo con materiales autoinstruccionales, Mi menú histórico realizadas en Ciencias Sociales, así como el desarrollo de guías de ejercicios y exposiciones, tanto en Matemáticas como Ciencias Naturales, permitieron que esta competencia, se mantuviera relativamente

44 Instituto de Investigación y Evaluación Educativas y Sociales / Universidad Pedagógica Nacional Francisco Morazán

(2) artículos de la Revista Electrónica Paradigma del Instituto de Investigación y Evaluación Educativas y Sociales de la Universidad Pedagógica Nacional Francisco Morazán, se comparten bajo términos de la Licencia Creative Commons: Se permite que otros puedan dinguna manera ni se pueden utilizar comercialmente. 
estable en porcentajes, luego de la intervención realizada, sin embargo, comparativamente, se encontró una disminución en porcentaje, con un $38 \%$.

La competencia de Busco información adicional sobre los temas, en el Pretest a juicio de los estudiantes, No ha sido desarrollada en ninguna de las tres asignaturas de su plan de estudio,(25\%) en el Pretest aplicado, aunque se puede observar, que en la opinión de otra parte de los estudiantes encuestados, en las 3 asignaturas, han visualizado, el desarrollo de esta competencia $(24 \%)$, se requieren estudios adicionales para determinar por qué los estudiantes señalan que no poseen esta competencia pero otra parte del grupo, tiene una percepción distinta.

En el Postest, con un porcentaje relativamente bajo de $22 \%$, los estudiantes señalaron que en las tres asignaturas, han adquirido medianamente esta competencia, este dato ocupó el segundo lugar, por la idea que, esta competencia todavía no ha sido desarrollada en los estudiantes, con un $20 \%$, al realizar la comparación, puede percibirse que particularmente esta competencia, no tiene un claro afincamiento en el grupo de competencias procedimentales, desarrolladas en los estudiantes de octavo grado del CIIE. Relaciono mi entorno con los temas estudiados, según los resultados del Pretest aplicado, con un porcentaje de $29 \%$, seguido por la idea de que en Ciencias Sociales y Ciencias Naturales, son las dos asignaturas que mejor han posibilitado, la adquisición de esta competencia, sin embargo, se encontró que con un mismo porcentaje, otro grupo de estudiantes, señaló que no posee esta competencia (21\%). En el Postest que se aplicó, los resultados mostraron que, un grupo importante de estudiantes (22 en total de 74) manifestó que esta competencia se ha desarrollado en las tres asignaturas.

La competencia de Comunico oralmente mis ideas, según los resultados del Pretest, en todas las asignaturas se ha desarrollado esta competencia, con un $24 \%$, En el Postest empleado, los resultados obtenidos, señalan que incrementó el dominio de esta competencia, en las tres asignaturas investigadas, con un $32 \%$, pudiendo inferirse que, las estrategias realizadas durante la implementación, motivaron 
a los estudiantes de octavo grado, a fortalecer esta competencia. Algunas estrategias desarrolladas en el experimento como Noche científica familiar, P.I.N, la Historia como un cuento animado, Bingo matemático, Mi primera investigación científica, etc. Contribuyeron seguramente a mejorar, el porcentaje obtenido en esta competencia.

La sexta competencia procedimental fue Elaboro informes de trabajo, se encontró que en las tres asignaturas estudiadas resultaron con el más alto índice de frecuencia y porcentaje, de $24 \%$, por otro lado, en el Postest, las asignaturas de Ciencias Sociales y Naturales resultaron con el porcentaje más alto de $35 \%$, este dato es consistente con las estrategias desarrolladas de manera particular en estas dos asignaturas, donde casi de manera tradicional, en el CIIE se han propiciado los espacios académicos, para que los estudiantes aprendan y mejoren su expresión escrita, mediante la elaboración de distintos documentos en el tercer nivel de educación básica. Interpreto dibujos y gráficas fue la séptima competencia propuesta, en esta competencia, la clase de matemáticas alcanzó el nivel más alto en el Pretest aplicado, con un $45 \%$. Comparativamente con las anteriores, esta competencia alcanzó un porcentaje relativamente alto, en el Postest, en cambio, resultó que las 3 asignaturas con un $32 \%$ han podido desarrollar esta competencia, nuevamente este resultado, permite afirmar que la implementación de las estrategias en estas clases, determinó o influyó en el resultado obtenido.

Otra competencia fue Registrar observaciones a través de dibujos y gráficas, los resultados muestran que los estudiantes afirmaron, no poseer esta competencia en ninguna de las asignaturas con un porcentaje de $32 \%$, en el Postest, a pesar de que los resultados anteriores mostraron que, los estudiantes eran capaces de interpretar las gráficas y dibujos presentados en documentos o producto de su propia elaboración, hace falta todavía emplear éstas en proceso de registro de observaciones, ello implica, que los estudiantes no utilizan el lenguaje simbólico para hacer sus anotaciones u observaciones de fenómenos o situaciones estudiadas, prefiriendo quizás, el lenguaje escrito y oral para cumplir con estas tareas, el porcentaje logrado en el Postest fue de 37\%. Es necesario, realizar otras estrategias que pudieran promover el desarrollo de esta competencia.

46

Instituto de Investigación y Evaluación Educativas y Sociales / Universidad Pedagógica Nacional Francisco Morazán

Los artículos de la Revista Electrónica Paradigma del Instituto de Investigación y Evaluación Educativas y Sociales de la Universidad Pedagógica Nacional Francisco Morazán, se comparten bajo términos de la Licencia Creative Commons: Se permite que otros puedan descargar las obras y compartirlas con otras personas, siempre y cuando se reconozca su autoría, pero no se pueden cambiar de $Y$ NC ND descargar las obras y compartirus con setras peisonas, sing 
La novena competencia procedimental fue Utilizar la computadora como herramienta para la resolución de problemas, Para esta competencia, la frecuencia más alta se logró en las asignaturas de Ciencias Sociales y Ciencias Naturales, con un 30\%, según el instrumento aplicado como Pretest, en el Postest, arrojó que las tres asignaturas han permitido en los estudiantes, el fortalecimiento de esta competencia procedimental con un porcentaje de $43 \%$. La Realización de proyectos multidisciplinares utilizando la computadora fue otra competencia procedimental en donde los resultados evidenciaron que alcanzó el porcentaje más alto en las asignaturas de Ciencias Sociales y Ciencias Naturales, con un $28 \%$, en el Postest, estas mismas asignaturas, alcanzaron el mismo posicionamiento con un leve incremento del $30 \%$, pero también las tres asignaturas en conjunto, lograron el mismo porcentaje, sin dudas, el nuevo elemento en este resultado es la clase de matemáticas, tal vez la implementación de las estrategias realizadas con el uso de la computadora, potenciaron que los estudiantes, ubicaran esta competencia desarrollada en estas asignaturas.

La competencia de Utilizar enciclopedias y buscadores de la web para hacer investigaciones, en el Pretest a criterio de los estudiantes consultados, en las asignaturas de Ciencias Sociales y Ciencias Naturales, con un promedio bastante alto casi del $50 \%$, seguido con una frecuencia considerable de 21 estudiantes que señalaron que, en las tres asignaturas consideran tener esta competencia, en el Postest, en cambio, en primer lugar, las 3 asignaturas resultaron con un porcentaje importante de $46 \%$, seguido por las asignaturas de Ciencias Sociales y Naturales con un $34 \%$, este resultado muestra la aplicación de estrategias didácticas, como Investigación bibliográfica, el Afiche personalizado, Mural del juego de valores, Noche científica familiar, prácticas de laboratorio, elaboración de maquetas y otras, contribuyeron a que los estudiantes pudieran valorar esta competencia en las 3 asignaturas que fueron objeto de intervención.

La selección apropiada de materiales y equipos para realizar una demostración fue la duodécima competencia establecida, los resultados mostraron que esta capacidad alcanzó mayor porcentaje $(25 \%)$ en todas las asignaturas, indicando que se ha desarrollado en 
las tres objeto de esta investigación, este dato estuvo seguido por las asignaturas de Ciencias Naturales y Sociales casi con un mismo porcentaje.

En el Postest, con un porcentaje bastante significativo (41\%) evidenció que para los estudiantes de octavo grado, esta competencia procedimental fue alcanzada en las tres asignaturas, aunque de manera consistente, en las clases de Ciencias Sociales y Ciencias Naturales, se logró un porcentaje considerable, señalando entonces que, en este resultado, la asignatura de matemáticas fue incluida en la valoración de los estudiantes, el conjunto de competencias procedimentales se cierra con el Manejo de objetos e instrumentos de laboratorio. La frecuencia más alta del Pretest (39.5\%) se logró en la asignatura de Ciencias Naturales, siendo necesario que se alcance en las otras asignaturas, en donde se manipula también objetos de laboratorio, en el sentido menos tradicional del término.

En el Postest, resultó que las tres asignaturas lograron un porcentaje similar $(40.5 \%)$ al alcanzado en el Pretest solo por la asignatura de Ciencias Naturales; interesante es que en ambos instrumentos, la frecuencia de respuestas es la misma; 30 estudiantes apuntaron primero esta competencia en Ciencias Naturales y en esta misma cantidad señalaron que en las tres asignaturas es posible identificar esta habilidad de manejar objetos e instrumentos de laboratorio.

En síntesis, los resultados del Pretest mostraron que no hubo diferencias significativas en las distintas competencias incluidas en el grupo Procedimental, de igual forma, en el Postest, no se lograron establecer diferencias significativas entre los dos grupos participantes, el experimental y el control, tal vez sea necesario revisar la cantidad de estrategias desarrolladas o el período de tiempo de la intervención, para establecer las condiciones que pudieron determinar que entre los grupos no hubiera diferencias estadísticamente significativas. No obstante, es importante analizar los resultados obtenidos para conocer las competencias procedimentales que poseen los estudiantes de octavo grado, sugiriendo luego a los directivos y docentes del CIIE, las estrategias que deben continuar potenciándose para lograr aprendizajes significativos, la tabla 2 evidencia este resultado. 
Desarrollar estrategias didácticas para la adquisición de competencias investigativas en estudiantes de octavo grado del Centro de Investigación e Innovación Educativas

Tabla 2

Resultados del postest en anova en las competencias procedimentales

\begin{tabular}{|c|c|c|c|}
\hline $\begin{array}{l}\text { Competencias } \\
\text { Procedimentales }\end{array}$ & & $\mathbf{F}$ & Sig. \\
\hline $\begin{array}{l}\text { Doy juicios de valor sobre los temas } \\
\text { estudiados }\end{array}$ & $\begin{array}{l}\text { Entre grupos/Dentro } \\
\text { de grupos }\end{array}$ & 1.205 & .276 \\
\hline Analizo textos escritos y orales & $\begin{array}{l}\text { Entre grupos/Dentro } \\
\text { de grupos }\end{array}$ & 3.339 & .072 \\
\hline $\begin{array}{l}\text { Busco información adicional sobre } \\
\text { los temas }\end{array}$ & $\begin{array}{l}\text { Entre grupos/Dentro } \\
\text { de grupos }\end{array}$ & .771 & .383 \\
\hline $\begin{array}{l}\text { Relaciono mi entorno con los temas } \\
\text { desarrollados }\end{array}$ & $\begin{array}{l}\text { Entre grupos/Dentro } \\
\text { de grupos }\end{array}$ & 1.476 & .228 \\
\hline Comunico oralmente mis ideas & $\begin{array}{l}\text { Entre grupos/Dentro } \\
\text { de grupos }\end{array}$ & 3.901 & .052 \\
\hline Elaboro informes de trabajo & $\begin{array}{l}\text { Entre grupos/Dentro } \\
\text { de grupos }\end{array}$ & .963 & .330 \\
\hline Interpreto dibujos y gráficas & $\begin{array}{l}\text { Entre grupos/Dentro } \\
\text { de grupos }\end{array}$ & .493 & .485 \\
\hline $\begin{array}{l}\text { Utilizo la computadora como } \\
\text { herramienta para la resolución de } \\
\text { problemas }\end{array}$ & $\begin{array}{l}\text { Entre grupos/Dentro } \\
\text { de grupos }\end{array}$ & .299 & .586 \\
\hline $\begin{array}{l}\text { Realizo proyectos multidisci- } \\
\text { plinares utilizando la computadora }\end{array}$ & $\begin{array}{l}\text { Entre grupos/Dentro } \\
\text { de grupos }\end{array}$ & 1.645 & .204 \\
\hline $\begin{array}{l}\text { Utilizo enciclopedias y buscadores } \\
\text { de la web para hacer } \\
\text { investigaciones }\end{array}$ & $\begin{array}{l}\text { Entre grupos/Dentro } \\
\text { de grupos }\end{array}$ & .029 & .866 \\
\hline $\begin{array}{l}\text { Selecciono apropiadamente los } \\
\text { materiales y equipos para realizar } \\
\text { una demostración }\end{array}$ & $\begin{array}{l}\text { Entre grupos/Dentro } \\
\text { de grupos }\end{array}$ & .123 & .727 \\
\hline $\begin{array}{l}\text { Manejo objetos e instrumentos de } \\
\text { laboratorio }\end{array}$ & $\begin{array}{l}\text { Entre grupos/Dentro } \\
\text { de grupos }\end{array}$ & 3.097 & .083 \\
\hline
\end{tabular}

Finalmente, en las competencias actitudinales, los resultados tanto del Pretest como del Postest aplicados a octavo grado, evidenciaron que todas las competencias, sin excepción alguna, están desarrolladas 
en estos estudiantes de manera simultánea en las tres asignaturas del estudio, no obstante, entre las competencias actitudinales, puede observarse en la tabla 3, que algunas de esas competencias lograron posiciones más altas, tanto en el Pretest como en el Postest, destacándose en particular la de aceptar sugerencias de sus compañeros y seguir indicaciones en el cumplimiento de asignaciones, dos competencias esenciales para el desempeño académico de los estudiantes, cuidando que en trabajos futuros se potencie más el trabajo de argumentación de respuestas por parte de los estudiantes.

\section{Tabla 3}

Resultados del pretest y postest en las competencias actitudinales

\begin{tabular}{lcc}
\hline & Pretest & Postest \\
\hline $\begin{array}{l}\text { Acepto las sugerencias y/o aportes que me hacen mis } \\
\text { compañeros y compañeras }\end{array}$ & $65.8 \%$ & $68.9 \%$ \\
Argumento mis respuestas & $34.2 \%$ & $35.1 \%$ \\
Colaboro con mi equipo cumpliendo mis asignaciones & $69.7 \%$ & $70.3 \%$ \\
Sigo instrucciones para realizar mis trabajos & $82.9 \%$ & $82.4 \%$ \\
Participo en actividades dentro y fuera del aula & $55.3 \%$ & $59.5 \%$ \\
Muestro curiosidad por cosas nuevas & $53.9 \%$ & $48.6 \%$ \\
\hline
\end{tabular}

Sin embargo, estadísticamente tampoco se lograron diferencias significativas entre los grupos control y experimental, la tabla número 4 afirma este resultado:

\section{Tabla 4}

Resultados del postest en anova en las competencias actitudinales

\begin{tabular}{lccc}
\hline \multicolumn{2}{l}{ Competencias Conceptuales } & F & Sig. \\
\hline $\begin{array}{l}\text { Acepto las sugerencias y/o } \\
\begin{array}{l}\text { aportes que me hacen mis } \\
\text { compañeros y compañeras }\end{array}\end{array}$ & $\begin{array}{c}\text { Entre grupos/Dentro } \\
\text { de grupos }\end{array}$ & 2.378 & .127 \\
Argumento mis respuestas & $\begin{array}{c}\text { Entre grupos/Dentro } \\
\text { de grupos }\end{array}$ & 1.460 & .231 \\
\hline
\end{tabular}

50 Instituto de Investigación y Evaluación Educativas y Sociales / Universidad Pedagógica Nacional Francisco Morazán

(i) Los artículos de la Revista Electrónica Paradigma del Instituto de Investigación y Evaluación Educativas y Sociales de la Universidad Pedagógica Nacional Francisco Morazán, se comparten bajo términos de la Licencia Creative Commons: Se permite que otros puedan descargar las obras y compartirlas con otras personas, siempre y cuando se reconozca su autoría, pero no se pueden cambiar de hinguna manera ni se pueden utilizar comercialmente. 
Desarrollar estrategias didácticas para la adquisición de competencias investigativas en estudiantes de octavo grado del Centro de Investigación e Innovación Educativas

\begin{tabular}{lccc}
\hline $\begin{array}{l}\text { Colaboro con mi equipo } \\
\text { cumpliendo mis asignaciones }\end{array}$ & $\begin{array}{c}\text { Entre grupos/Dentro } \\
\text { de grupos }\end{array}$ & .427 & .516 \\
$\begin{array}{l}\text { Sigo instrucciones para realizar mis } \\
\text { trabajos }\end{array}$ & $\begin{array}{c}\text { Entre grupos/Dentro } \\
\text { de grupos }\end{array}$ & 2.379 & .127 \\
$\begin{array}{l}\text { Participo en actividades dentro y } \\
\text { fuera del aula }\end{array}$ & $\begin{array}{c}\text { Entre grupos/Dentro } \\
\text { de grupos }\end{array}$ & 2.186 & .144 \\
$\begin{array}{l}\text { Muestro curiosidad por cosas } \\
\text { nuevas }\end{array}$ & $\begin{array}{c}\text { Entre grupos/Dentro } \\
\text { de grupos }\end{array}$ & .213 & .646 \\
\hline
\end{tabular}

\section{Conclusiones}

1. Los resultados mostrados en la aplicación del Pretest y Postest, luego de la intervención realizada, evidencian que los estudiantes de octavo grado poseen competencias conceptuales, procedimentales y actitudinales, sin embargo, el nivel desarrollado para cada una es diferente. En las competencias conceptuales se encontró que la competencia de Proponer problemas en clase ha sido más desarrollada en la asignatura de Ciencias Sociales y Plantear soluciones a problemas en la clase de Matemáticas.

Las competencias de Hago preguntas sobre los temas desarrollados, Participo activamente en los temas desarrollados y Propongo soluciones a problemas surgidos en clase, se lograron desarrollar con porcentajes significativos en las tres asignaturas en estudio, mientras que las competencias Manejo más contenido que el abordado en clase, Explico conceptos o términos sobre los temas e Invento nuevos problemas, no fueran alcanzadas por los estudiantes de octavo grado, a pesar de la intervención realizada. En las competencias procedimentales, los estudiantes participantes señalaron que no poseen dichas habilidades propuestas en ninguna de las tres asignaturas, estas competencias son: Doy juicios de valor sobre los temas estudiados y Registro observaciones a través de dibujos y gráficas, en el resto de competencias procedimentales los estudiantes indicaron que 
sí alcanzaron estas competencias, particularmente a través de las asignaturas de Ciencias Sociales y Ciencias Naturales seguido por matemáticas.

Las competencias actitudinales, tanto en el Pretest como en el Postest, evidenciaron que los estudiantes de octavo grado cuentan con esas competencias, la diferencia encontrada fue que algunos casos se incrementaron el porcentaje del dominio manifestado. Investigaciones posteriores que puedan realizarse en la línea de las competencias investigativas pudieran enfatizar en el fortalecimiento de las competencias conceptuales y procedimentales, tomando como punto de partida los resultados obtenidos en este estudio.

2. Las estrategias desarrolladas durante la intervención fueron seleccionadas e implementadas en la mayoría de casos por los docentes de cada asignatura, sin embargo, en algunas semanas del período escolar, las mismas fueron desarrolladas por docentes en práctica profesional de la Universidad Pedagógica Nacional, debido a que el CIIE tiene entre sus funciones servir de centro de práctica a los estudiantes por egresar de este centro educativo.

Las estrategias fueron ejecutadas durante tres meses completos, el suministro de materiales y equipos para la realización de las estrategias fue en gran parte financiado por el proyecto de investigación, contando también con los recursos institucionales, de los docentes y de los practicantes de las asignaturas de Ciencias Sociales y Matemáticas, los resultados mostraron que no hubo diferencias estadísticamente significativas entre los grupos control y experimental, es decir, que la intervención realizada no constituyó el elemento determinante para lograr por un lado, que algunas competencias identificadas en el Pretest, que no habían sido desarrolladas, se alcanzaran luego de la intervención, así como lograr que las competencias propuestas en la investigación fueran desarrolladas en las tres asignaturas de manera más sistemática, es preciso entonces continuar con 
Desarrollar estrategias didácticas para la adquisición de competencias investigativas

en estudiantes de octavo grado del Centro de Investigación e Innovación Educativas

otros estudios que pudieran explicar, cuáles son las mejores condiciones para que los estudiantes obtengan mejores resultados, ya sea considerar el tiempo de la intervención, el número y tipo de estrategias aplicadas, los recursos y materiales empleados y los espacios físicos idóneos para desarrollar estrategias. Las competencias seleccionadas necesitan valorarse más y bajo otras circunstancias, en estudios de esta naturaleza.

3. En cuanto a las estrategias didácticas más significativas, puede concluirse, por las observaciones realizadas durante el período de intervención, que en la clase de matemáticas, Pluto y el hueso, Práctica de laboratorio con el programa Geógebra y el Bingo fueron las estrategias que más motivación causaron entre los estudiantes, promoviendo que mediante el juego, pueden lograrse aprendizajes significativos. Estas estrategias fueron adaptadas por el docente titular y el practicante respectivo, logrando convertir actividades cotidianas en estrategias didácticas, que en el caso del Bingo y Pluto, pueden adaptarse a otras asignaturas obteniendo mayor interés y mostrando que pueden existir muchos caminos para el aprendizaje de las matemáticas. En la clase de Ciencias Naturales, las estrategias de la Noche familiar científica y la hoja de cálculo JOMAR fueron las actividades que propiciaron mayor dinamismo en los jóvenes, particularmente la noche familiar científica, realizada en años anteriores, también permite el desarrollo de todo el proceso investigativo que culmina con la presentación oral de resultados de los estudiantes ante los miembros de su familia, quienes evalúan con un porcentaje de la nota total, las actividades realizadas por sus hijos e hijas. La hoja de cálculo JOMAR ofrece la oportunidad de aprender el tema de la conversión de unidades sin necesidad de memorizar fórmulas que seguramente serán olvidadas en el siguiente año escolar.

Estas dos estrategias fueron expresamente creadas por el docente de Ciencias Naturales del CIIE, mismas que han sido utilizadas en los diferentes cursos impartidos. En Ciencias Sociales se aplicaron una gama de estrategias, sin duda, las 
más creativas fueron $\mathrm{El}$ afiche personalizado, mi primera investigación científica y mi menú histórico. Estas estrategias también son inéditas y promueven la aplicación práctica de los conocimientos adquiridos en el estudio de las unidades de esta asignatura, las evidencias fotográficas que se anexan al informe, muestran el nivel organizativo, el talento y la creatividad de los estudiantes de octavo grado, que animadamente participaron en todas las estrategias implementadas.

4. Las competencias seleccionadas para esta investigación representan un variado conjunto de habilidades y destrezas esperadas en los estudiantes de octavo grado. Dentro de las competencias conceptuales, se identificaron 3 que lograron ser alcanzadas en las tres asignaturas de la intervención (Hago preguntas sobre los temas desarrollados, Participo activamente en los temas desarrollados y Propongo soluciones a problemas surgidos en clase) mientras que las otras 5 competencias, tres de ellas no lograron alcanzar el dominio por parte de los estudiantes (Manejo más contenido que el abordado en clase, Explico conceptos o términos sobre los temas e Invento nuevos problemas) y 2 de ellas, se posicionaron en las asignaturas de Ciencias Sociales (Propongo problemas en clase). En Matemáticas (Planteo posibles soluciones para resolver problemas); en las competencias procedimentales 13 en total, dos de ellas no lograron alcanzarse (Doy juicios de valor sobre los temas estudiados y Registro observaciones a través de dibujos y gráficas) mientras que en 10 competencias, los estudiantes señalaron que se desarrollaron en todas las asignaturas: Analizo textos escritos y orales, Busco información adicional sobre los temas, Relaciono mi entorno con los temas desarrollados, Comunico oralmente mis ideas, Interpreto dibujos y gráficas, Utilizo la computadora como herramienta para la resolución de problemas, Realizo proyectos multidisciplinares utilizando la computadora, Utilizo enciclopedias y buscadores de la web para hacer investigaciones, Selecciono apropiadamente los materiales y equipos para realizar una demostración y Manejo objetos e

54 Instituto de Investigación y Evaluación Educativas y Sociales / Universidad Pedagógica Nacional Francisco Morazán Los artículos de la Revista Electrónica Paradigma del Instituto de Investigación y Evaluación Educativas y Sociales de la Universidad Pedagógica Nacional Francisco Morazán, se comparten bajo términos de la Licencia Creative Commons: Se permite que otros puedan descargar las obras y compris ninguna manera ni se pueden utilizar comercialmente. 
Desarrollar estrategias didácticas para la adquisición de competencias investigativas en estudiantes de octavo grado del Centro de Investigación e Innovación Educativas

instrumentos de laboratorio y solamente en una de las competencias, se visualizó lograda en Ciencias Sociales y Ciencias Naturales (Elaboro informes de trabajo). Resulta interesante el caso de las competencias actitudinales, ya que tanto en el Pretest como en el Postest las seis competencias establecidas se lograron desarrollar antes de la intervención y se mantuvieron luego de la experiencia realizada.

\section{Recomendaciones}

El Centro de Investigación e Innovación Educativas debe continuar con el desarrollo de estudios sistemáticos que ayuden a desarrollar competencias investigativas en sus estudiantes, los resultados obtenidos en esta investigación, señalan puntualmente las competencias, en particular las conceptuales, que deben seguirse potenciando en la implementación de estrategias didácticas permanentes en las diferentes asignaturas que conforman el Plan de estudios, independientemente del bachillerato que se pretenda estudiar. Ello implica asimismo, trabajar las competencias procedimentales y actitudinales, abriendo la posibilidad de incluir otras habilidades que se consideren pertinentes y necesarias para los estudiantes del centro.

\section{Referencias}

Balbo, J. (s/f). Formación en competencias investigativas, un nuevo reto de las universidades. Disponible en http:/ / www.ucv.ve/ fileadmin/user_upload/vrac/documentos/ Curricular_Documentos/Evento/Ponencias/Balbo_josefina.pdf

Centro de Investigación e Innovación Educativas. (2001). El contexto Interno (documento sin publicar). Honduras.

Estado de Honduras. (1982). Constitución de la República de Honduras. Tegucigalpa: Guaymuras. 
Sonia Patricia Guity López, José Alfonzo Mendoza Corrales

Íñiguez, F. (2014). El desarrollo de la competencia matemática en el aula de ciencias experimentales. Revista Iberoamericana de Educación/Revista Ibero-americana de Educação, vol. 67, núm. 2 (15/ 03/15), pp. 117-130

Muñoz, G., Federman, J., Quintero, J., Molina, M., Ancizar, R. (2005). Cómo desarrollar competencias investigativas en Educación. Colombia: Aula abierta.

Suazo, M; \& Moncada, G. (2001). La Investigación en Honduras. Honduras: Dirección de Investigación de la UPNFM.

Universidad Nacional de Educación a Distancia. (s/f). ¿Qué son estrategias didácticas? Disponible en https:/ / www.uned.ac.cr/ ac a d e mica / i mages / ceced/d ocs / Estaticos / contenidos_curso_2013.pdf 